\title{
Identification of filtration and migration parameters in the MATLAB calculation environment using numerical simulation of breakthrough curve and optimization methods
}

\author{
M. Okonska ${ }^{1}$, M. Marciniak ${ }^{1}$, M. Kaczmarek ${ }^{2}$ \\ $\&$ K. Kazimierska-Drobny ${ }^{2}$ \\ ${ }^{1}$ Adam Mickiewicz University, Poznan, Poland \\ ${ }^{2}$ Kazimierz Wielki University, Bydgoszcz, Poland
}

\begin{abstract}
Mathematical modelling and numerical simulations in the processes of groundwater's filtration and pollutant transport are dependent on the knowledge of hydrogeological parameters of a given medium. The authors were interested in the possibility of defining the value of hydrogeological parameters using the method of numerical optimization. So far the identification of the parameters appearing in the description of transport process has been conducted mostly using the method of trial and error. In the article the authors present their own method of determining filtration and migration parameters on the basis of the data registered during the column experiment using numerical optimization in MATLAB calculation environment. The procedure proved considerable convergence and not having many local minimums. Good fit of numerical models with experimental breakthrough curves was obtained. Moreover, the article presents the influence of particular hydrogeological parameters on the shape of breakthrough curve in MATLAB programme.
\end{abstract}

Keywords: filtration columns, breakthrough curves, MATLAB, numerical optimization, parameters of filtration and migration. 


\section{Introduction}

Mathematical modelling and numerical simulation of filtration processes of groundwater and pollutant transport in aquifers are currently an intensively developing branch of hydrogeological research. There is a growing number of specialized computer programmes which enable numerical simulation of the processes. The possibility of conducting simulation calculations is however dependent on the knowledge of numerical values of all hydrogeological parameters occurring in the mathematical description of filtration of groundwater and pollutant transport in a given model. The urgent task is to create new and effective methods of defining hydrogeological parameters values.

The authors of the article conducted a series of column experiments in laboratory conditions. As a result of the experiment we registered breakthrough curves showing variation of tracer concentration in time function. The processes of water filtration and substance migration through the ground sample which took place during the experiment were described using mathematical equations. In the mathematical model which was used to identify hydrogeological parameters, programming environment MATLAB and its inbuilt functions were used. Suggested identification procedure enabled to obtain the set of required parameters using a numerical optimization method.

\section{Experimental research}

Experimental research was conducted on the experimental workstation, in which three filtration columns with differing dimensions were constructed (fig.1). Columns were built from polymethyl methacrylate (PMMA), the material which ensures chemical neutrality in relation to liquids taking part in experiments.

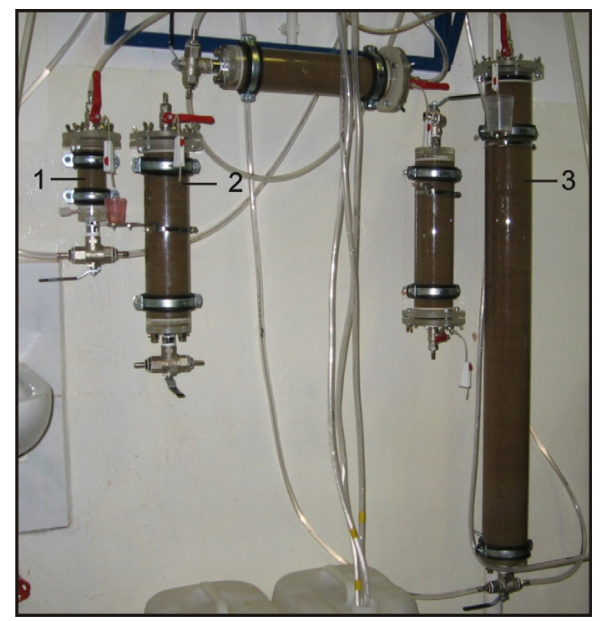

Figure 1: Filtration columns: 1 - length $10 \mathrm{~cm}$ and diameter $40 \mathrm{~mm}, 2$ length $30 \mathrm{~cm}$ and diameter $70 \mathrm{~mm}, 3$ - length $110 \mathrm{~cm}$ and diameter $80 \mathrm{~mm}$. 
Column experiment was made by filtrating the tracer dissolved in water through samples of coarse sand placed in the columns. The research involved experiments with two types of tracers: chloride ions $\mathrm{Cl}^{-}$treated as conservative tracer, undergoing advection and dispersion processes and lithium ions $\mathrm{Li}^{+}$ treated as sorbed tracer, undergoing processes of both advection, dispersion and sorption. All experiments were conducted in dynamic conditions, with a constant hydraulic gradient $I$. It was assumed that research was conducted in an oxidation environment. The tracer injection was planned according to a step method, i.e. according to the Heaviside unit step function (continual injection of the tracer) and impulse method, compatible with practical realisation of Dirac impulse function (short-term injection of a tracer), fig.2. Concentration of tracers was marked after filtration through the sand sample in order to draw their breakthrough curve. In the course of laboratory research three types of breakthrough curves were registered: upward-sloping curve, downward-sloping curve and impulse curve, fig.3. Each of the experiments on the laboratory workstation was conducted according to the same research procedure which was created in the course of earlier column research (Okonska [5]).
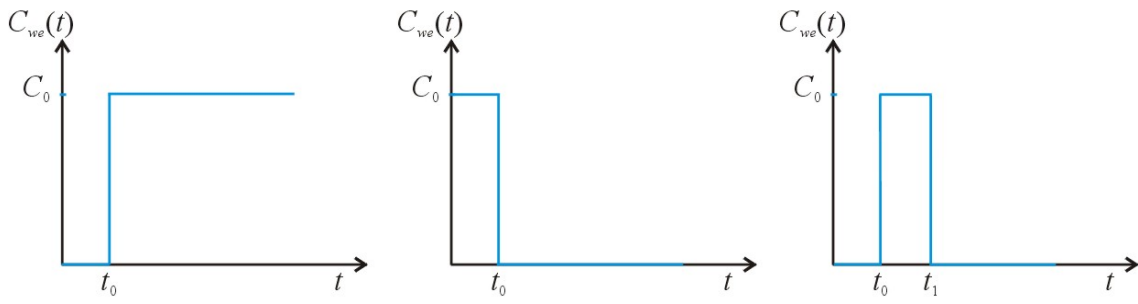

Figure 2: Identifying signals used in column experiments: increasing unit step, decreasing unit step, impulse.
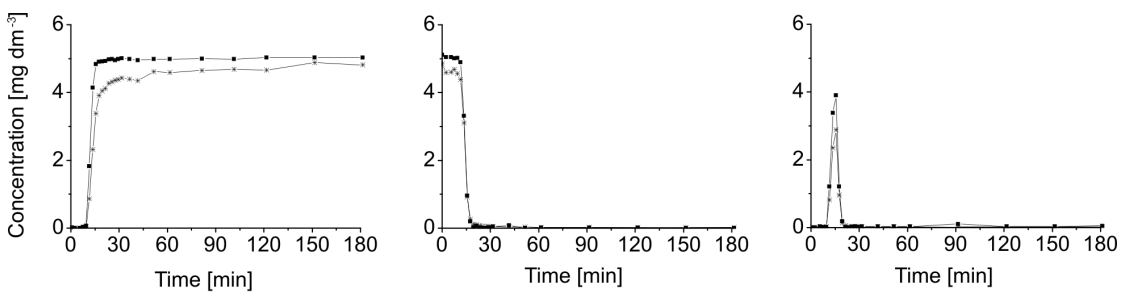

Figure 3: The graphs of tracer breakthrough curves registered during experiments in column 2. Squared lines - concentration for chloride ions $* 100$, star lines - concentration for lithium ions $* 1$.

The porosity of particular sand samples was estimated during column research, as a part of the hydrogeological parameters recognition process. Measurements of the hydraulic gradient and the filtration discharge were also conducted, which made it possible to determine the filtration parameters. Moreover, the temperature and electrolytical conductivity of the solutions were measured. 


\section{Mathematical description of filtration processes and tracer migration in filtration column}

Processes of transport in column tests are one-dimensional processes. The description of dimensional, one direction pollutant transport in grounds accompanied by simultaneous change of their density in time, for conservative tracers is limited to the description of advection and dispersion processes which in turn consists of molecular diffusion and mechanical dispersion. In case of sorbed tracer, mathematical transport model in the soil is extended by the sorption process. For the saturated porous medium, moderately and easily permeable, in filtration conditions based on Darcy's law, advection processes and mechanical dispersion connected with porosity and tortuosity of the medium are significant.

Advection-dispersion equations of one-direction mass transport in saturated porous materials, taking into account equilibrium sorption or linear irreversible kinetic sorption have respective form (Fetter [3]):

$$
\begin{gathered}
\frac{\partial C}{\partial t}=-\frac{v_{a}}{R} \frac{\partial C}{\partial x}+\frac{D}{R} \frac{\partial^{2} C}{\partial x^{2}}, \\
\frac{\partial C}{\partial t}=-v_{a} \frac{\partial C}{\partial x}+D \frac{\partial^{2} C}{\partial x^{2}}-\frac{\rho_{s}}{n} k_{1} C,
\end{gathered}
$$

where: $C$ - substance concentration $\left[\mathrm{mg} \mathrm{dm}^{-3}\right], v_{a}$ - average porous velocity (advection) $\left[\mathrm{m} \mathrm{s}^{-1}\right], x$ and $t-$ space and time variable [m], $[\mathrm{s}], D-$ hydromechanical dispersion coefficient $\left[\mathrm{m}^{2} \mathrm{~s}^{-1}\right], R$-retardation factor [-], $k_{1}-$ reaction rate constant $[-], \rho_{\mathrm{s}}-$ density of solid particles $\left[\mathrm{kg} \mathrm{m}^{-3}\right]$ and $n-$ total porosity [-].

In case of equilibrium sorption, there was considered Henry's linear model and Freudlich power model which have the form of:

$$
\begin{gathered}
R_{d}=1+\frac{\rho_{s}}{n} K_{d}, \\
R_{F}=1+\frac{\rho_{s} K_{F} N C^{N-1}}{n},
\end{gathered}
$$

where: $K_{d}-$ Henry's distribution coefficient, $K_{F}-$ Freudlich distribution coefficient, $N$ - exponent of Freudlich model.

The solution of equations (1) and (2) in a configuration appropriate for column test was obtained by assessing that on the upper boundary (as in the experiment) there are increasing, decreasing or impulse concentrations, with $C_{0}$ amplitude. The initial condition for the first and third cases were zero, and in decreasing condition it was $C_{0}$. In the lower ground sample base it was assumed that there is lack of diffusion transport, i.e.:

$$
\frac{\partial C}{\partial x}=0
$$


The equations of transport which take into account mechanisms of advection, dispersion and processes of sorption are square partial differential equations. Only in some special cases there are precise analytical solutions of the equations. Analytical solutions useful in column test simulation are characterized by poor convergence of solution series. Due to this, the solutions of simple and inverse problem related to column tests will be using only numerical solutions. The simulations will be conducted in calculation MATLAB environment in which partial differential equations (PDE) are solved through the function (solver) pdepe, which uses numerical method of finite elements. The pdepe function solves initial-boundary problems for parabolic and elliptic partial differential equations with one time variable $x$ and in case of parabolic equations with time variable $t$ in finite space and time intervals. In the procedure realized by pdepe we go from partial differential equations to ordinary differential equations (ODE) using strict space discretization of second rank, based on the nodes network defined by the user.

\section{Parametrical analysis of breakthrough curves}

In the course of the research parametrical analysis for all three types of input signal function was conducted. The analysis consisted of defining what is the influence of particular parameters of pollutant transport in ground medium on the shape of breakthrough curve. The solution to the equations (1) and (2) was obtained by using inbuilt numerical procedures in MATLAB programme, based on the finite elements method. The analysis of breakthrough curves sensitivity was conducted for the parameters of one of the filtration columns which was used in laboratory research (column 2).

Examples of concentration evolution for different values of effective porosity $n_{e}$ are presented in figure 4 . The values of other parameters used for the simulation are constant and are as follows: dispersivity $\alpha_{L}=0,001 \mathrm{~m}$, ground sample length $L=0,3 \mathrm{~m}$, hydraulic gradient $I=0,1 \mathrm{~m} / \mathrm{m}$, total porosity $n=0,3$, density of solid particles $\rho_{s}=2,65 \mathrm{~kg} / \mathrm{dm}^{3}$, initial concentration of tracer $C_{0}=10 \mathrm{mg} / \mathrm{dm}^{3}$. Other assessed parameters: effective coefficient of molecular diffusion $D_{M}=1,5 \cdot 10^{-9} \mathrm{~m}^{2} / \mathrm{s}$, permeability coefficient of the researched ground $k=1 \cdot 10^{-5} \mathrm{~m} / \mathrm{s}$, sorption parameters $K_{d}=0,01, K_{f}=0,01, N=0,9, k_{1}=10^{-7}$. The increase of effective porosity causes decrease of advection velocity and slower transport of the substance through the column. The influence of effective porosity on the steepness of breakthrough curves and impulse shape can also be observed.

In figure 5 there are compared breakthrough curves for three, physically justified dispersion values $\alpha_{L}=0,0001 \mathrm{~m}, \alpha_{L}=0,001 \mathrm{~m}$ and $\alpha_{L}=0,01 \mathrm{~m}$. The values of other parameters are as previously, effective porosity $n_{e}=0,3$. Dispersivity changes slope of breakthrough curves. The higher dispersivity value, the less steep is the slope. In case of impulse signal dispersivity causes shifts in time and change of impulse shape and maximum height.

The influence of sorption parameters is presented in the figures $6,7,8$ and 9 . 

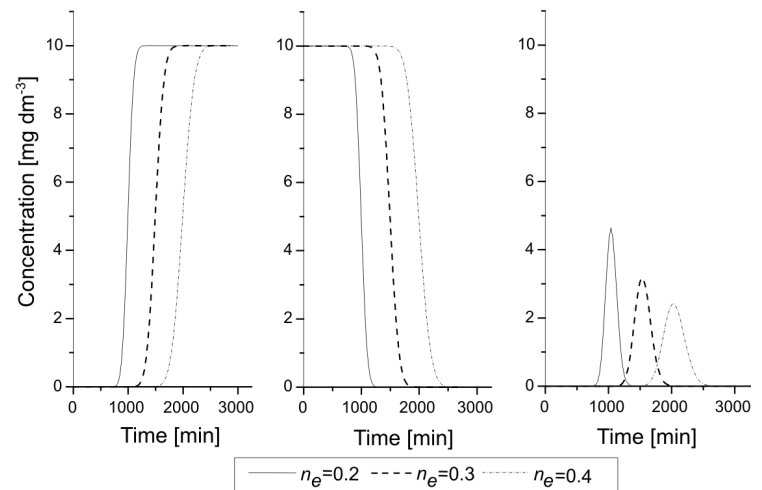

Figure 4: The evolution of breakthrough curves for different values of effective porosity $n_{e}$.
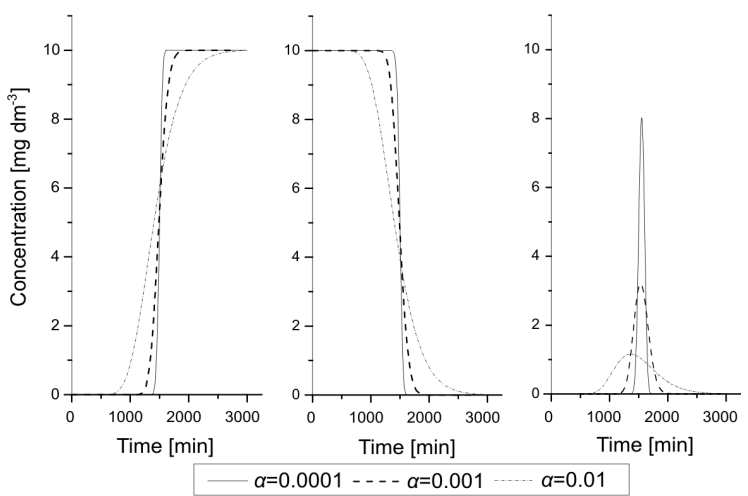

Figure 5: The evolution of breakthrough curves for different values of dispersivity $\alpha$.
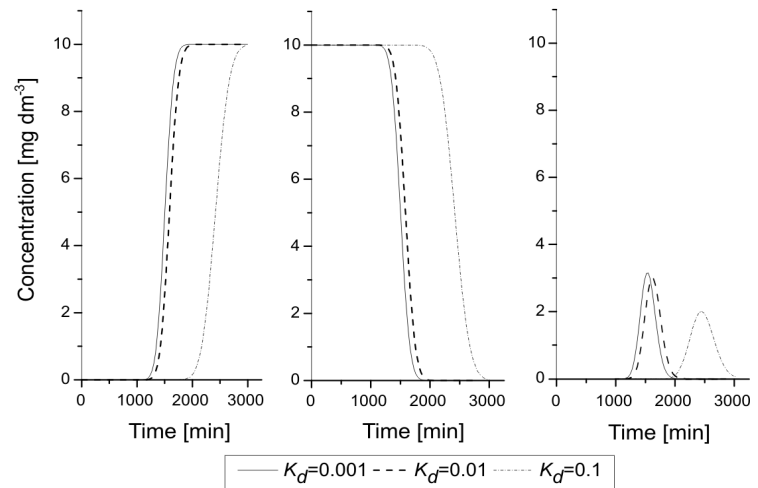

Figure 6: The evolution of breakthrough curves for different values of Henry's distribution coefficient $K_{d}$. 

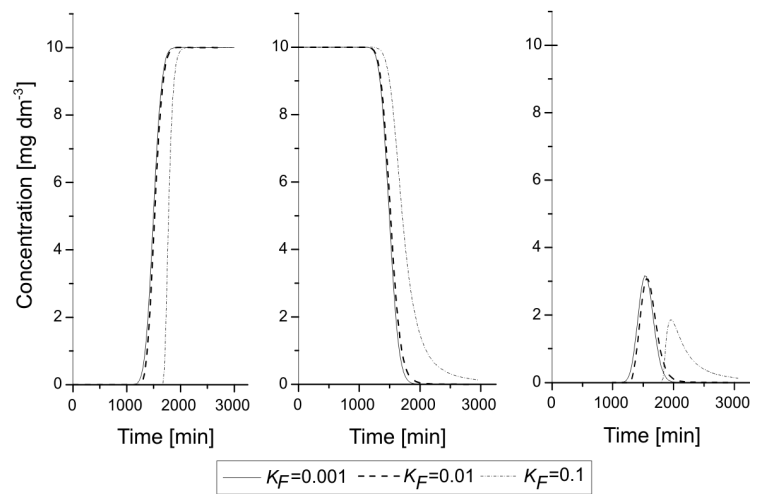

Figure 7: The evolution of breakthrough curves for different values of Freudlich distribution coefficient $K_{F}$.
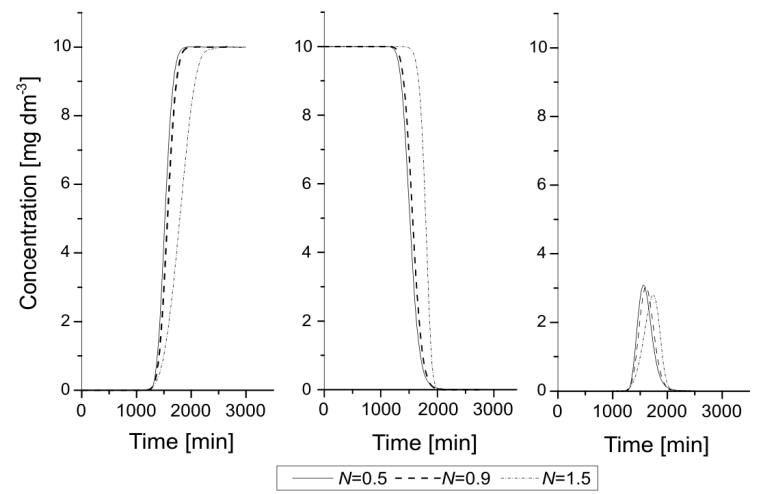

Figure 8: The evolution of breakthrough curves for different values of exponent of Freudlich model $N$.
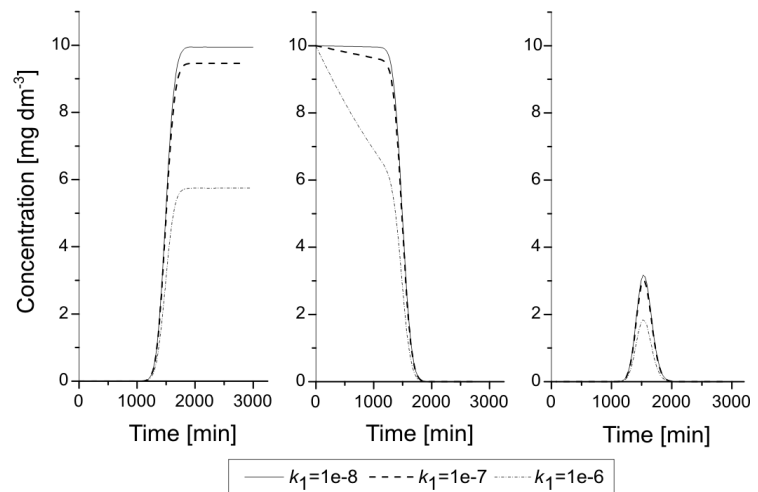

Figure 9: The evolution of breakthrough curve for different values of firstorder reaction rate constant $k_{1}$. 
From presented simulations one can assume that the increase of distribution coefficient $K_{d}$ or $K_{F}$ results in parallel shift of breakthrough curve, i.e. delay in pollutant migration through the sample. There are observed small changes during incoming substances for low coefficient value. The increase of distribution coefficient causes the shift in impulses and lowering maximums. It does not result in the loss of breakthrough curve symmetry for Henry's distribution coefficient $K_{d}$. The exponent $N$ in Freudlich model does not change the time after which the pollutant appears on the column's boundary, but changes the shape of breakthrough curve slope. As the exponent $N$ increases, there is also an increase of breakthrough curve steepness [3]. In case of impulse signal the increase of exponent $N$ causes slight decrease and shift of maximum. It can be concluded from observation that exponent $N$ influences breakthrough curve steepness, i.e. intensity of pollutant transport through the filtration column. The increase of first-order reaction rate constant $k_{1}$ does not cause the delay of substance transport, but it influences the final value of concentration at the end of column. According to expectations, the increase of constant $k_{1}$ is accompanied by the decrease of concentration of pollutants after going through the column. Theoretically, this model does not provide possibility for the decrease of concentration, which can be observed from equation (2). The results with decreasing impulse lose therefore physical sense. In case of impulse signal, the constant $k_{1}$ results in decrease of impulse value maximums, but it does not change their symmetry.

\section{The method of numerical optimization in the identification of filtration and transport parameters}

Identification of model parameters, including procedures which using experimental data aim to find parameter model value, is classified into a broader class of problems called solving inverse problem. The procedures of parameter identification are usually searching for the best fit of theoretical curves (model solutions) to the data obtained in the experiment. In order to do that various approaches are used - from trial and error procedure to using advanced computer optimization methods and so called artificial intelligence methods. Trial and error procedure uses knowledge and experience of the person using the method and generally is not effective and not precise, especially in case of searching for a bigger number of parameters. Hence, there is observed large interest in development of optimization methods where effectiveness is less dependent on subjective factors, although the element of ability to select effective for a given problem optimization method is still very significant. The literature which is available to a limited extent involves knowledge on the selection of optimization methods and each of the problems requires separate studies.

The procedure of filtration parameters identification which was created, was divided into two stages. Each of the stage was realized taking into account appropriate data obtained during column test and appropriate migration model. During the first stage of the research, there were only identified on the basis of conservative tracers breakthrough curves (ions $\mathrm{Cl}^{-}$) parameters of advection and 
dispersion: effective porosity $n_{e}$ and dispersivity $\alpha_{L}$. In the second stage there were taken into account defined in the first stage parameters $n_{e}$ and $\alpha_{\mathrm{L}}$, and identified on the basis of sorbed tracers breakthrough curves (ions $\mathrm{Li}^{+}$) sorption parameters: distribution coefficient $K_{d}$, Freudlich model parameters $K_{F}$ and $N$ or reaction rate constant of kinetics model $k_{1}$.

The model of migration process was that there was constant velocity of the flow through the column forced by constant hydraulic gradient, domination of mechanical dispersion over diffusion and three models of sorption: Henry's equilibrium linear model, Freudlich equilibrium non-linear model and kinetic linear model of irreversible sorption.

Optimization procedures which are to identify parameters were defined in MATLAB environment equipped with optimization packet Optimization Toolbox. There were used inbuilt functions dedicated to solving non-linear optimization problems. In order to estimate the parameters there was conducted solution of inverse problem, while in checking estimation results there was used solution of simple initial-boundary problem with zero initial condition, boundary conditions of Dirichlet and Newmann types, respectively for input and output of the column. There were taken into account three types of tracer injection. The solution was obtained through inbuilt pdepe function. The proper one for the convergence number of spatial elements and time steps was estimated using sensitivity research. Due to the fact that frequency of sample taking in column experiments could be significantly lower that required number of steps for securing convergence of pdepe procedure, the intervals between measures were made more dense by the method of linear interpolation with additional points (from 10 to 20 points). To calculate error function ef in the form of the sum of difference square of the values of measured concentration $C$ and obtained from simulation $C_{m}$ were only analysed concentrations in the moments of measuring $t_{k}$ :

$$
e f=\sum_{k=1}^{N}\left[C\left(t_{k}\right)-C_{m}\left(\mathbf{A}, t_{k}\right)\right]^{2},
$$

where: $C$ - experimental result for the moment $t_{k}, C_{m}$ - the value of concentration defined from mathematical model for the moment $t_{k}, \mathbf{A}-$ set of identified parameters.

Minimization of the error function (inverse problem solution) was obtained using inbuilt in optimization MATLAB packet function of lsqcurvefit, which is the implementation of the trust region method (Coleman and Li [2]). The method searches for the minimum of function which approximates error function by its Taylor series expansion with accuracy to square term and assumes limited independent variable step with appropriate scaling of the variable. As a result a following problem is solved:

$$
\min \left\{\frac{1}{2} S^{T} H S+S^{T} g \text { that }\|D S\| \leq \Delta\right\},
$$


where: $g$ - gradient of error function, $H$ - error function Hessian, $S$ minimization step vector, $S^{T}$ - transposed vector in minimization step, $D-$ diagonal scaling matrix, $\Delta$-parameter defining confidence region.

Scaling in each step is realised in order to eliminate unnecessary for gradient methods results of large differences in sensitivity of error function for independent variables. The choice of optimization procedure, criteria of minimization ending, options of displaying the results, etc., are made by defining arguments of optimset function which specification precedes calling function lsqcurvefit. Parameters of ending iteration minimization process must be settled during numerical experiments which can use data obtained through simulation.

On the selected examples we checked the possibility of a unique solution of the inverse problem, analysing dependence of error function on the values of identified parameters, defined in the optimization procedure.

\section{Results}

As a result of the research there were registered breakthrough curves for conservative ions (chloride) and sorbed ions (lithium). Each of the registered curves was used to identify transport parameters. The results of column research which were obtained and calculations of identification parameters are presented in table 1 .

Table 1: $\quad$ Results of hydrogeological parameters identification.

\begin{tabular}{|c|c|c|c|c|c|c|c|c|c|c|c|c|}
\hline \multicolumn{5}{|c|}{ Experiment } & \multicolumn{8}{|c|}{ Model } \\
\hline$L$ & $C_{0 C l}$ & $C_{0 L i}$ & $n$ & $k$ & $n_{e}$ & $\alpha$ & $K_{d}$ & $R_{d}$ & $K_{F}$ & $N$ & $R_{F}$ & $k_{1}$ \\
\hline$[\mathrm{m}]$ & {$\left[\mathrm{mg} / \mathrm{dm}^{3}\right]$} & {$\left[\mathrm{mg} / \mathrm{dm}^{3}\right]$} & {$[-]$} & {$[\mathrm{m} / \mathrm{s}]$} & {$[-]$} & {$[\mathrm{m}]$} & {$\left[\mathrm{g} / \mathrm{cm}^{3}\right]$} & - & {$\left[\mathrm{g} / \mathrm{cm}^{3}\right]$} & - & - & {$\left[\mathrm{g} / \mathrm{cm}^{3}\right]$} \\
\hline \multirow{3}{*}{0.1} & 488.1 & 4.36 & \multirow{3}{*}{0.38} & $\begin{array}{c}2.78 \mathrm{E}- \\
03\end{array}$ & 0.64 & 0.00370 & 0.03390 & 1.15 & 0.00130 & 3.12140 & 1.40 & 0.000017 \\
\hline & 490.5 & 4.35 & & $\begin{array}{c}2.40 \mathrm{E}- \\
03\end{array}$ & 0.40 & 0.00250 & 0.00420 & 1.02 & 0.12390 & 0.07280 & 1.01 & \\
\hline & 485.6 & 4.42 & & $\begin{array}{c}2.70 \mathrm{E}- \\
03 \\
\end{array}$ & 0.54 & 0.00030 & 0.00320 & 1.01 & 0.00520 & 1.01200 & 1.02 & 0.000111 \\
\hline \multirow{3}{*}{0.3} & 516.3 & 4.84 & \multirow{3}{*}{0.37} & $\begin{array}{c}1.81 \mathrm{E}- \\
03\end{array}$ & 0.41 & 0.00240 & 0.00353 & 1.02 & 0.00013 & 4.88550 & 2.34 & 0.000033 \\
\hline & 511.3 & 4.85 & & $\begin{array}{c}1.53 \mathrm{E}- \\
03 \\
\end{array}$ & 0.40 & 0.00190 & 0.00170 & 1.01 & 0.03640 & 0.09740 & 1.00 & \\
\hline & 511.3 & 4.80 & & $\begin{array}{c}1.69 \mathrm{E}- \\
03 \\
\end{array}$ & 0.40 & 0.00200 & 0.00280 & 1.01 & 0.00430 & 1.03600 & 1.02 & 0.000084 \\
\hline \multirow{3}{*}{1.0} & 507.4 & 4.71 & \multirow{3}{*}{0.38} & $\begin{array}{c}2.05 \mathrm{E}- \\
03\end{array}$ & 0.41 & 0.00240 & 0.04450 & 1.19 & 0.00810 & 2.25710 & 1.55 & 0.000032 \\
\hline & 507.4 & 4.71 & & $\begin{array}{c}2.06 \mathrm{E}- \\
03 \\
\end{array}$ & 0.38 & 0.00370 & 0.00650 & 1.03 & & & & \\
\hline & 507.4 & 4.74 & & $\begin{array}{c}2.17 \mathrm{E}- \\
03\end{array}$ & 0.37 & 0.00500 & 0.00570 & 1.02 & 0.00690 & 1.00400 & 1.03 & 0.000028 \\
\hline
\end{tabular}

Explanation: $L$ - length of sand sample, $C_{0}$ - concentration of tracer in a solution, $k$ - permeability coefficient, $n$ - total porosity, $n_{\mathrm{e}}-$ effective porosity, $\alpha$ - dispersivity, $K_{d}$ - distribution coefficient for a linear sorption, $R_{d}$ - Henry's retardation factor, $K_{F}$ - Freudlich distribution coefficient, $N$ - exponent of Freudlich model, $R_{F}-$ Freudlich retardation factor, $k_{1}-$ reaction rate constant. 


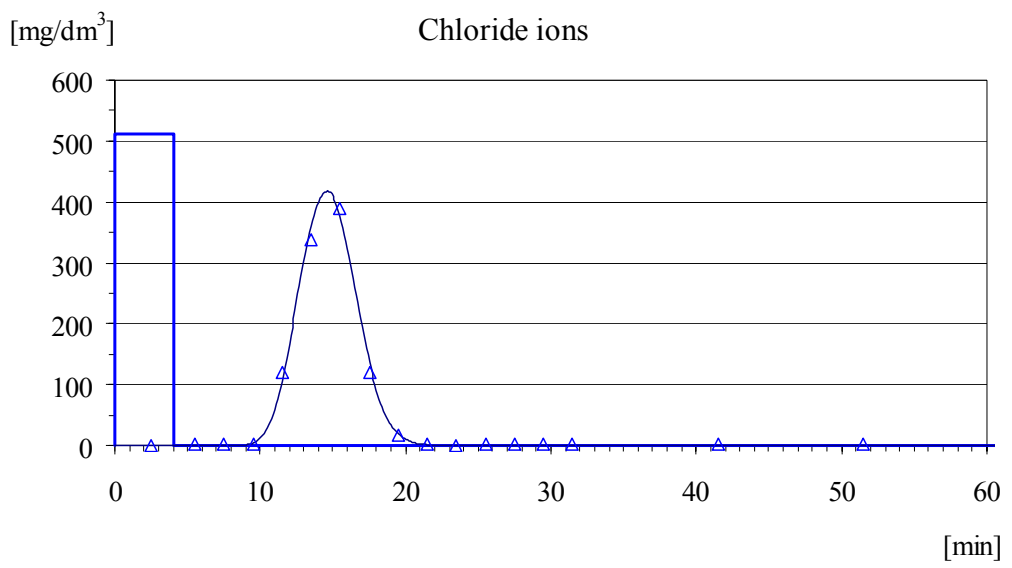

$\left[\mathrm{mg} / \mathrm{dm}^{3}\right]$

Lithium ions

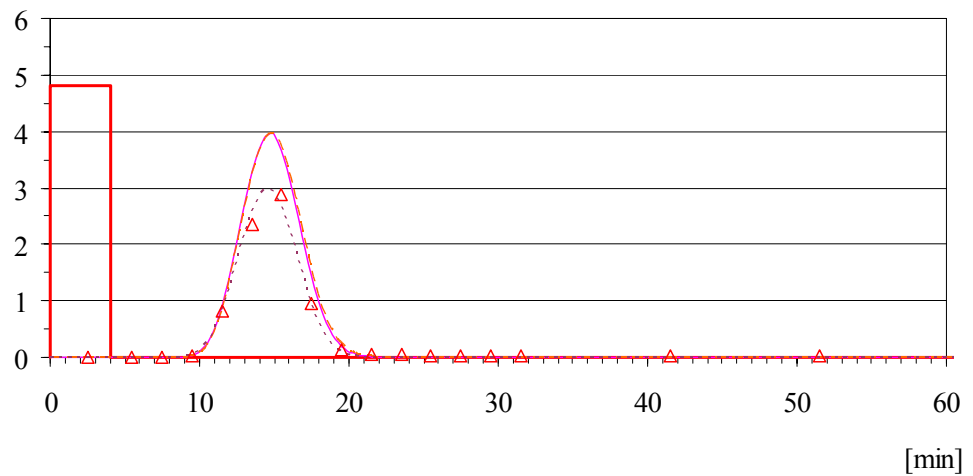

Figure 10: Comparison of impulse breakthrough curves of tracers. Explanation in the text.

The example of numerical curves fit, calculated in MATLAB programme for experimental tracer breakthrough curve present graphs for column 2 shown in figure 10. In the graphs, in the case of conservative tracer, a solid line marked a breakthrough curve calculated in the MATLAB programme, which was obtained in the course of identification. In the case of the sorbed tracer, the solid line marked a breakthrough curve calculated in the MATLAB programme in the course of identification conducted for the sorption according to Henry's model. The semi-line marked the results of calculations for the sorption according to Freudlich model. Whereas the dotted line marked results of calculations for nonequilibrium sorption with first-order reaction kinetics.

\section{Conclusions}

The identification procedure which was constructed, using gradient method of confidence region implemented in MATLAB, proved good convergence and 
little dependence on the selection of estimation starting point and lack of a large number of local minimums. It means a significant likelihood of an optimization procedure.

The end of the optimization procedure took place on the basis of obtaining an error function minimum according to the given criterion of minimum threshold for the changes in error function or changes of identified parameters vector.

For the most of the breakthrough curves a qualitatively good fit of models was obtained including identified parameters.

Obtained parameter values of advection-dispersion transport $n_{e}$ and $\alpha_{L}$ could be assumed as correct despite some differences for various types input signals.

In the case of sorbed tracer (lithium ions) the fit of experimental and calculation breakthrough curves is correct. Probably, one could obtain a better fit by selecting another sorption model, change of boundary conditions or a better quality of data obtained in the course of experiment.

\section{References}

[1] Appelo C.A.J. \& Postma D., Geochemistry, groundwater and pollution, A.A. Balkema: Rotterdam, Brookfield, 1999.

[2] Coleman T. F. \& Li Y., An interior trust region approach for nonlinear minimization subject to bounds. SIAM J. Optimization, 6, pp. 418-445, 1996.

[3] Fetter C.W., Applied Hydrogeology, Prentice Hall: Upper Saddle River, 2001.

[4] Marciniak M. \& Okonska M., The identification of hydrogeological parameters on the basis of the column experiment modelling. From data gathering and groundwater modelling to integrated management, Instituto Geologico y Minero de Espana, Serie: Hidrogeologia y aguas subterraneas, 21: Madrid, pp. 421-427, 2006.

[5] Okońska M., Identyfikacja parametrów migracji zanieczyszczeń w porowatym ośrodku hydrogeologicznym metodq modelowania eksperymentu kolumnowego, Geologos 9, Monographiae 3, Bogucki Wydawnictwo Naukowe: Poznań, 2006 (in Polish).

[6] Pratap R., Matlab 7 dla naukowców i inżynierów, PWN: Warszawa, 2007 (in Polish). 